Electronic Supplementary Information (ESI).

\title{
Bio-inspired hybrid fluorescent ligands for the FK1 domain of FKBP52
}

List of Contents:

S1. Protein Preparation.

S2. Pseudopeptide Concentration.

S2. X-ray Crystallography Data Collection.

S3. Compound Characterisation.

S11. References.

MALDI-TOF mass spectrometry analyses were performed in the ion positive reflector mode on an ABI Voyager DE-Pro MALDI-TOF mass spectrometer (Applied Biosystems) using as matrix a saturated solution of $\alpha$-cyano-4-hydroxy-cinnamic acid (CHCA) in $\mathrm{CH}_{3} \mathrm{CN}: \mathrm{H}_{2} \mathrm{O}: \mathrm{CF}_{3} \mathrm{COOH}$ (50:50:0.1). Experiments were performed at the IBPS-UPMC.

\section{Protein preparation:}

Protein concentration for FKBP-FK1 was determined by UV-Vis absorbance @ 280 nm using a nanodrop setup and aided by the presence of two Tryptophan residues. The extinction coefficient was calculated $(15,470)$ using expasy. FKBP-FK2 concentration was determined using the BSA assay as reported previously. ${ }^{1}$ 


\section{PseudoPeptide concentration:}

Lyophilised peptides were weighed on a high accuracy microbalance. The peptides were dissolved in water, sonicated and then an equal volume of 2x PBS was added. The concentration was determined by UV-Vis Absorbance on a nanodrop 2000c spectrophotometer in a $1 \mathrm{~cm}$ pathlength cuvette using an epsilon of $3400 @ 331 \mathrm{~nm}$.

\section{Crystallography:}

\section{Crystallographic data collection}

\begin{tabular}{|c|c|}
\hline & hFKBP52-FK1/Compound 1 \\
\hline X-ray source & PX2-25032018 \\
\hline Wavelength $(\AA)$ & 0.976 \\
\hline Temperature (K) & 100 \\
\hline Unit-cell parameters $\left(\AA{ }^{\circ},{ }^{\circ}\right)$ & $\begin{array}{l}a=b=52.92, c=237.68, \alpha=\beta= \\
90, \gamma=120.0\end{array}$ \\
\hline Space group & $\mathrm{P}_{6} 22$ \\
\hline Resolution limits $^{\dagger}(\AA ̊)$ & $45.86-2.30(2.44-2.30)$ \\
\hline Number of observations $^{\dagger}$ & $194498(28708)$ \\
\hline Number of unique reflections & $9583(1440)$ \\
\hline R-meas ${ }^{\dagger}(\%)$ & $12.7(240.5)$ \\
\hline Completeness $^{\dagger}(\%)$ & $99.6(97.5)$ \\
\hline $\mathrm{I} / \sigma \dagger$ & $15.55(0.99)$ \\
\hline $\mathrm{CC}(1 / 2)$ & $99.9(78.0)$ \\
\hline
\end{tabular}

Refinement

\begin{tabular}{|l|l|}
\hline & hFKBP52-FK1/Compound 1 \\
\hline $\begin{array}{l}\text { Number of non-hydrogen atoms } \\
\text { (protein/other) }\end{array}$ & $1017 / 55$ \\
\hline R/R & \\
\hline R.M.S.D. Bonds $(\AA) /$ angles $\left({ }^{\circ}\right)$ & $24 / 28$ \\
\hline $\begin{array}{l}\text { Average temperature factors } \\
\text { (protein/other) }\end{array}$ & $0.01 / 1.76$ \\
\hline
\end{tabular}

${ }^{\dagger}$ Values in parentheses refer to the highest resolution shell.

\section{Accession number}

Coordinates and structure factors have been deposited in the Protein Data Bank with accession number 6RCY. 


\section{Pseudopeptide Characterisation:}

All peptides are as the TFA salts following cleavage and HPLC purification.<smiles>CN(C)c1cccc2c(S(=O)(=O)N3CCCCC3C(=O)N[C@@H](CCc3ccccc3)C(=O)N[C@@H](CCCNC(=N)N)C(=O)O)cccc12</smiles>

Compound 1. Dansyl-Pip-hPhe-Arg-OH: m/z: MALDI-TOF $[\mathrm{M}+\mathrm{H}]^{+}: 680.47$ (Found), 680.32 (Calc.). Semi-Prep HPLC: Xbridge; $20-60 \%$ B over $10 \mathrm{~min} . \mathrm{Rt}=7.22 \mathrm{~min}$. Analytical HPLC: Proto $200 ; 20-80 \%$ B over $10 \mathrm{~min} . \mathrm{Rt}=6.60 \mathrm{~min}$.<smiles>CN(C)c1cccc2c(S(=O)(=O)N3CCCCC3C(=O)N[C@@H](Cc3ccccc3)C(=O)N[C@@H](CCCNC(=N)N)C(=O)O)cccc12</smiles>

Compound 2. Dansyl-Pip-Phe-Arg-OH: m/z: MALDI-TOF $[\mathrm{M}+\mathrm{H}]^{+}: 666.53$ (Found), 666.31 (Calc.). Semi-Prep HPLC: Xbridge; $20-60 \%$ B over 10 min. Rt = 7.18 min. Analytical HPLC: Proto 200; 20 $-80 \%$ B over $10 \mathrm{~min} . \mathrm{Rt}=6.48 \mathrm{~min}$. 


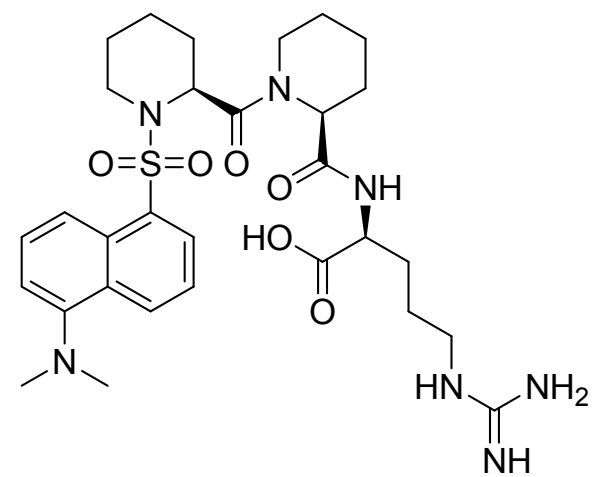

Compound 3. Dansyl-Pip-Pip-Arg-OH: m/z: MALDI-TOF [M+H] $]^{+}: 630.63$ (Found), 630.31 (Calc.). Semi-Prep HPLC: Xbridge; $20-60 \%$ B over 10 min. Rt = 4.90 min. Analytical HPLC: Proto 200; 20 $-80 \%$ B over $10 \mathrm{~min} . \mathrm{Rt}=5.00 \mathrm{~min}$.<smiles>CN(C)c1cccc2c(S(=O)(=O)N3CCCCC3C(=O)NC3(C(=O)N[C@@H](CCCNC(=N)N)C(=O)O)CCCC3)cccc12</smiles>

Compound 4. Dansyl-Pip-Acpa-Arg-OH: m/z: MALDI-TOF $[\mathrm{M}+\mathrm{H}]^{+}: 630.70$ (Found), 630.31 (Calc.). Semi-Prep HPLC: Xbridge; $20-60 \%$ B over 10 min. Rt $=4.99$ min. Analytical HPLC: Proto $200 ; 20-80 \%$ B over $10 \mathrm{~min} . \mathrm{Rt}=5.41 \mathrm{~min}$.<smiles>CN(C)c1cccc2c(S(=O)(=O)N3CCCCC3C(=O)N3Cc4ccccc4C[C@H]3C(=O)N[C@@H](CCCNC(=N)N)C(=O)O)cccc12</smiles> 
Compound 5. Dansyl-Pip-Tic-Arg-OH: m/z: MALDI-TOF $[\mathrm{M}+\mathrm{H}]^{+}: 678.85$ (Found), 678.31 (Calc.). Semi-Prep HPLC: Xbridge; $20-70 \%$ B over 10 min. Rt = 5.21 min. Analytical HPLC: Proto 200; 20 $-80 \%$ B over $10 \mathrm{~min} . \mathrm{Rt}=5.71 \mathrm{~min}$.

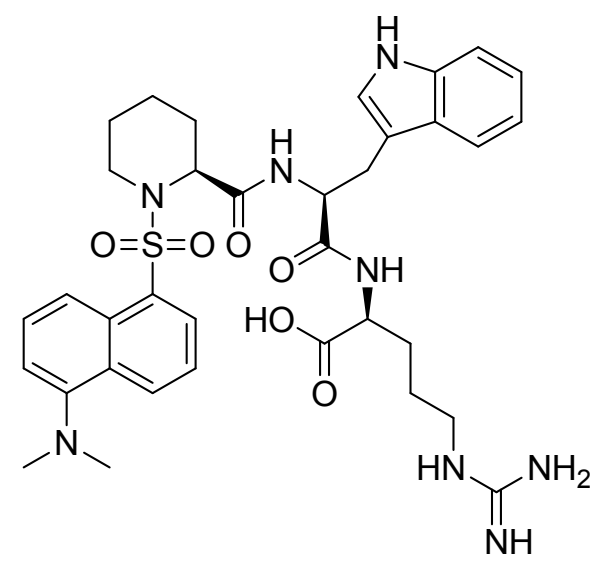

Compound 6. Dansyl-Pip-Trp-Arg-OH: m/z: MALDI-TOF [M+H] $]^{+} 705.80$ (Found), 705.32 (Calc.). Semi-Prep HPLC: Xbridge; $20-70 \%$ B over $10 \mathrm{~min} . \mathrm{Rt}=5.59 \mathrm{~min}$. Analytical HPLC: Proto 200; 20 $-80 \% \mathrm{~B}$ over $10 \mathrm{~min} . \mathrm{Rt}=5.91 \mathrm{~min}$.

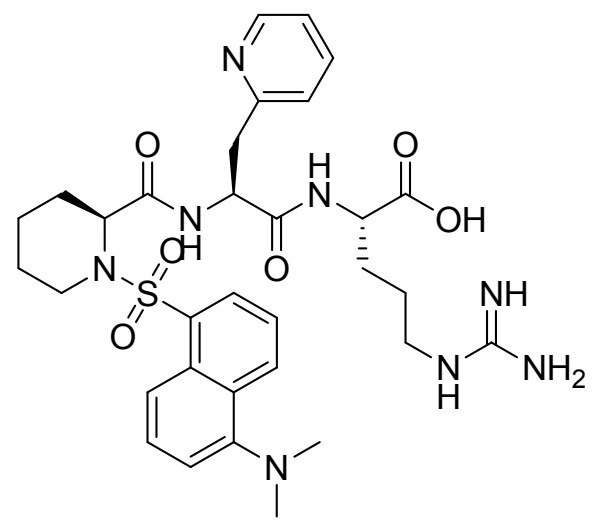

Compound 7. Dansyl-Pip-2-pyridylAla-Arg- $\mathrm{OH}$ : m/z: MALDI-TOF [M+H]+: 667.88 (Found), 667.30 (Calc.). Semi-Prep HPLC: Xbridge; $20-60 \%$ B over $10 \mathrm{~min}$. Rt $=6.21 \mathrm{~min}$. Analytical HPLC: Proto 200; $20-80 \%$ B over $10 \mathrm{~min} . \mathrm{Rt}=4.99 \mathrm{~min}$. 


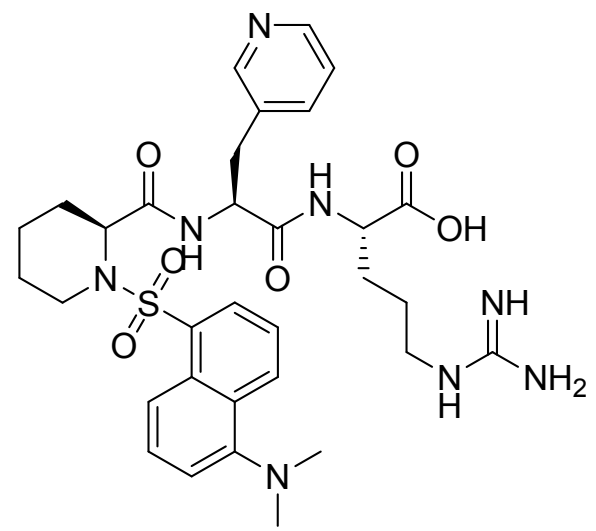

Compound 8. Dansyl-Pip-3-pyridylAla-Arg- $\mathrm{OH}$ : m/z: MALDI-TOF [M+H]+: 667.88 (Found), 667.30 (Calc.). Semi-Prep HPLC: Xbridge; $20-60 \%$ B over 10 min. Rt $=6.1$ min. Analytical HPLC: Proto $200 ; 20-80 \%$ B over $10 \min . \mathrm{Rt}=5.01 \mathrm{~min}$.

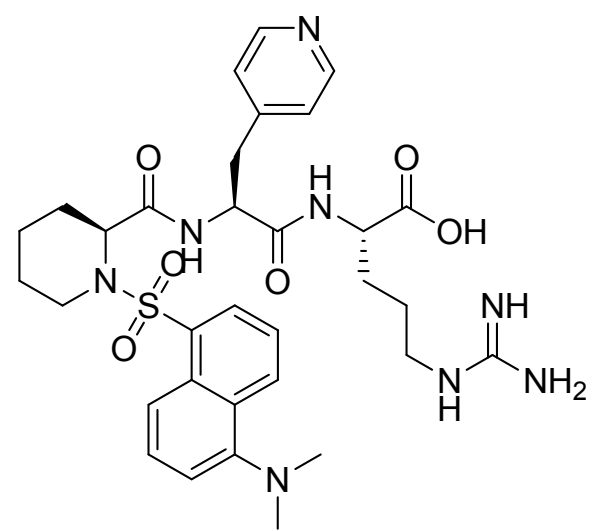

Compound 9. Dansyl-Pip-4-pyridylAla-Arg-OH: m/z: MALDI-TOF [M+H] $]^{+}: 667.64$ (Found), 667.30 (Calc.). Semi-Prep HPLC: Xbridge; $20-60 \%$ B over 10 min. Rt $=7.31$ min. Analytical HPLC: Proto $200 ; 20-80 \%$ B over $10 \mathrm{~min} . \mathrm{Rt}=5.41 \mathrm{~min}$.<smiles>CN(C)c1cccc2c(S(=O)(=O)N3CCCC[C@H]3C(=O)NC(C(=O)N[C@@H](CCCNC(=N)N)C(=O)O)C(C)(C)C)cccc12</smiles> 
Compound 10. Dansyl-Pip-tLeu-Arg-OH: m/z: MALDI-TOF $[\mathrm{M}+\mathrm{H}]^{+}: 632.77$ (Found), 632.32 (Calc.). Semi-Prep HPLC: Xbridge; $20-60 \%$ B over 10 min. Rt $=7.00$ min. Analytical HPLC: Proto $200 ; 20-80 \%$ B over 10 min. $\mathrm{Rt}=5.76 \mathrm{~min}$.

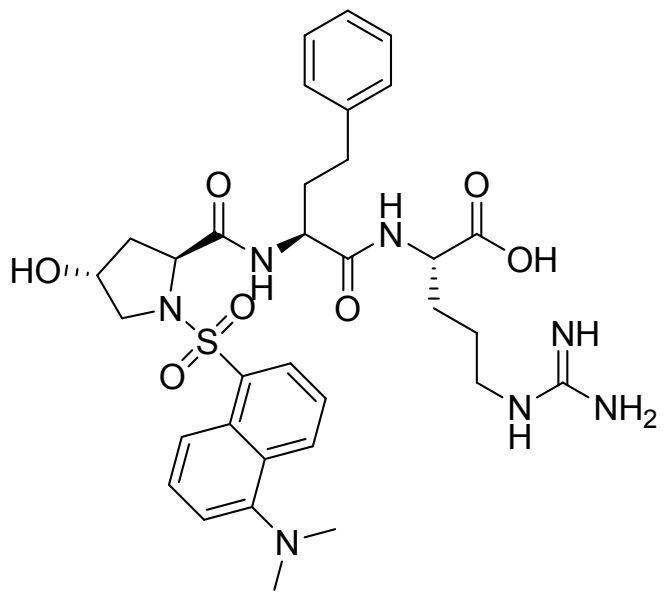

Compound 11. Dansyl-4R-Hyp-hPhe-Arg- $\mathrm{OH}: \mathrm{m} / \mathrm{z}$ : MALDI-TOF $[\mathrm{M}+\mathrm{H}]^{+}: 682.80$ (Found), 682,30 (Calc.). Semi-Prep HPLC: Xbridge; $20-60 \%$ B over 10 min. Rt $=7.18$ min. Analytical HPLC: Proto $200 ; 20-80 \%$ B over $10 \mathrm{~min} . \mathrm{Rt}=4.63 \mathrm{~min}$.

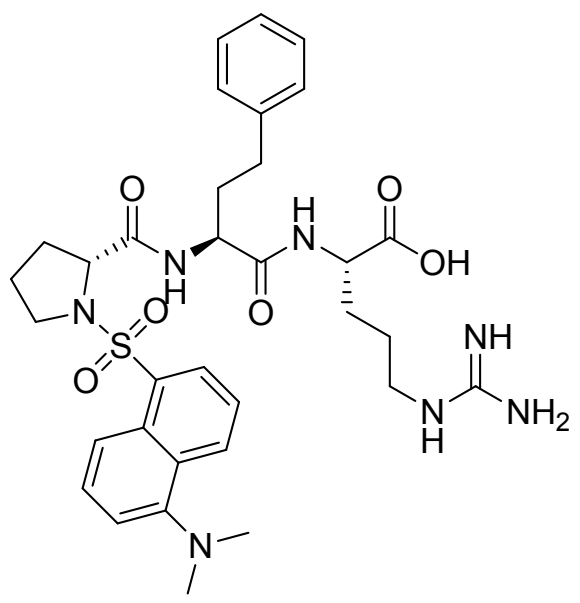

Compound 12. Dansyl-D-Pro-hPhe-Arg-OH: m/z: MALDI-TOF $[\mathrm{M}+\mathrm{H}]^{+} 666.66$ (Found) 666.31, (Calc.). Semi-Prep HPLC: Xbridge; $20-60 \%$ B over $10 \mathrm{~min} . \mathrm{Rt}=5.51 \mathrm{~min}$. Analytical HPLC: Proto $200 ; 20-80 \%$ B over $10 \min . \mathrm{Rt}=6.11 \mathrm{~min}$. 


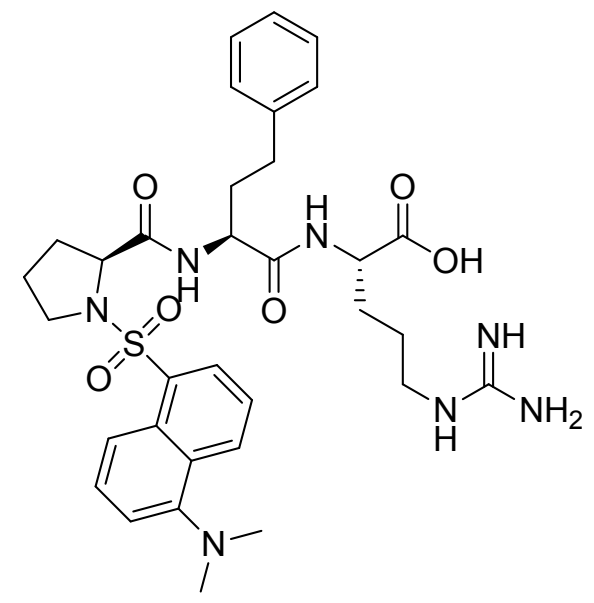

Compound 13. Dansyl-L-Pro-hPhe-Arg-OH: m/z: MALDI-TOF $[\mathrm{M}+\mathrm{H}]^{+} 666.64$ (Found), 666.31 (Calc.). Semi-Prep HPLC: Xbridge; $25-50 \%$ B over 10 min. Rt $=4.97$ min. Analytical HPLC: Proto $200 ; 20-80 \%$ B over $10 \min . \mathrm{Rt}=6.08 \mathrm{~min}$.

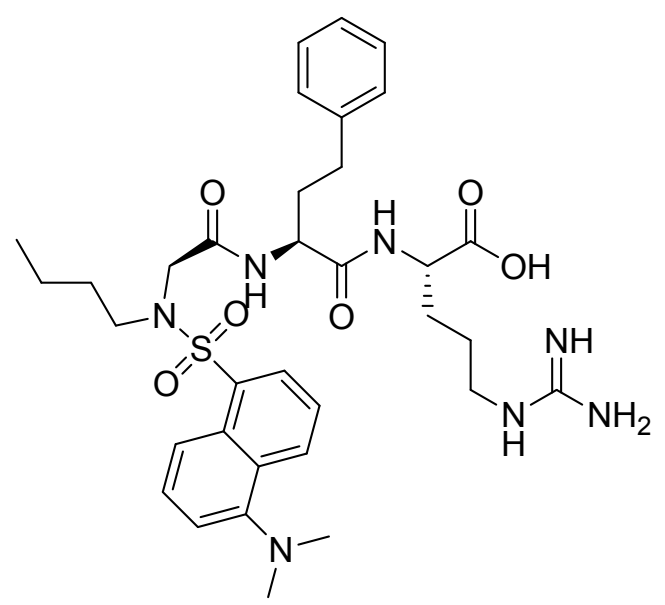

Compound 14. Dansyl- $n B u$-Gly-hPhe-Arg-OH: m/z: MALDI-TOF $[\mathrm{M}+\mathrm{H}]^{+}: 682.62$ (Found), 682.34 (Calc.). Semi-Prep HPLC: Xbridge; $20-60 \%$ B over 10 min. Rt $=6.78$ min. Analytical HPLC: Proto $200 ; 20-80 \%$ B over 10 min. Rt $=7.14 \min$. 


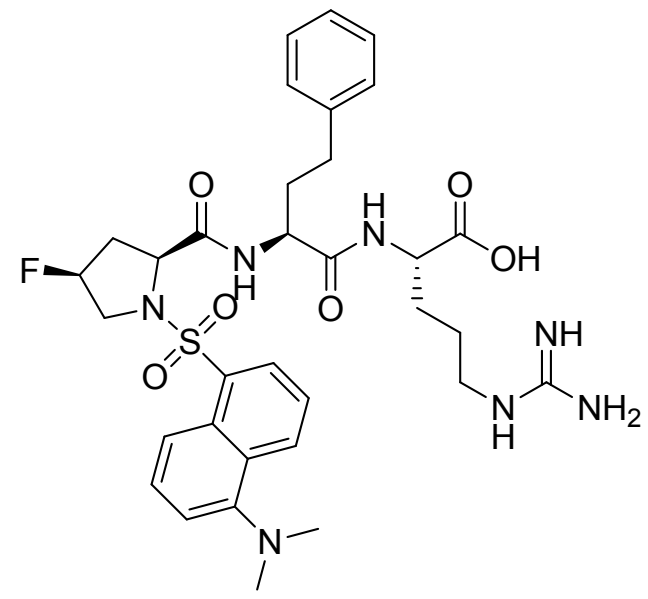

Compound 15 Dansyl-2S,4S-flp-hPhe-Arg-OH: m/z: MALDI-TOF [M+H] $]^{+}: 684.62$ (Found), 684.30 (Calc). Semi-Prep HPLC: Xbridge; $20-50 \%$ B over 10 min. Rt $=5.94$ min. Analytical HPLC: Proto $200 ; 20-80 \%$ B over 10 min. $\mathrm{Rt}=6.00 \mathrm{~min}$.

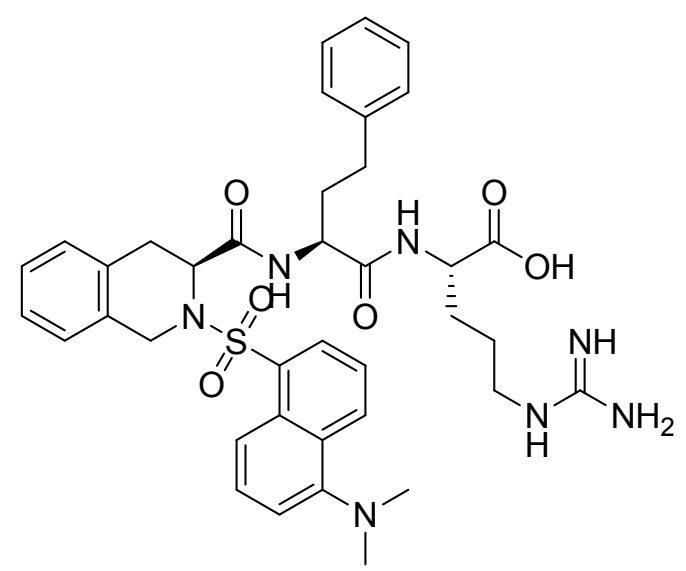

Compound 16. Dansyl-Tic-hPhe-Arg- $\mathrm{OH}: \mathrm{m} / \mathrm{z}$ : MALDI-TOF $[\mathrm{M}+\mathrm{H}]^{+}: 728.67$ (Found), 728.32 (Calc). Semi-Prep HPLC: Xbridge; $20-60 \%$ B over 10 min. Rt $=7.13$ min. Analytical HPLC: Proto $200 ; 20-80 \%$ B over $10 \mathrm{~min} . \mathrm{Rt}=7.30 \mathrm{~min}$. 


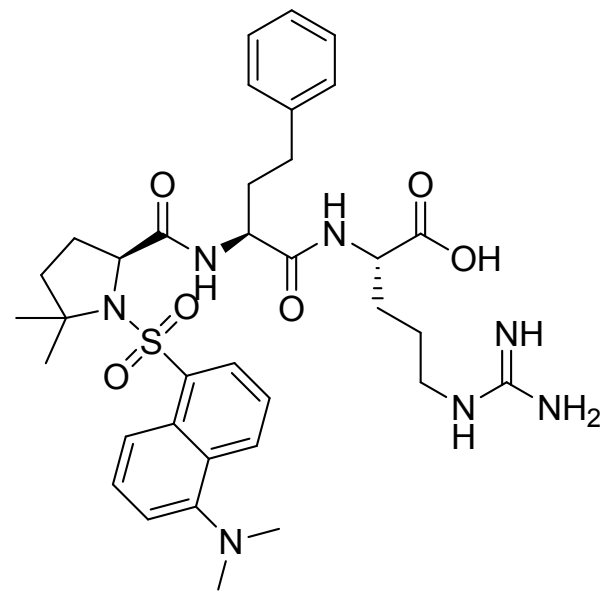

Compound 17. Dansyl-dmP-hPhe-Arg- $\mathrm{OH}: \mathrm{m} / \mathrm{z}$ : MALDI-TOF $[\mathrm{M}+\mathrm{H}]^{+}: 694.70$ (Found), 694.34 (Calc.). Semi-Prep HPLC: Xbridge; $20-50 \%$ B over 10 min. Rt $=7.47$ min. Analytical HPLC: Proto $200 ; 20-80 \%$ B over $10 \min . \mathrm{Rt}=6.16 \mathrm{~min}$.

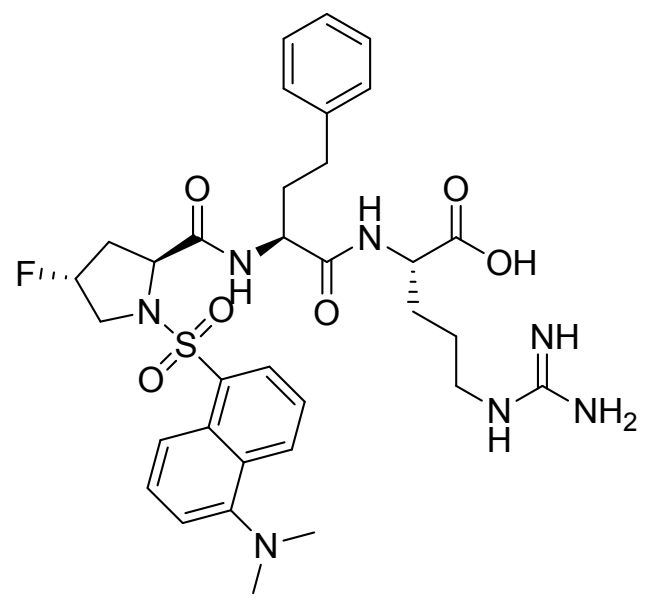

Compound 18 Dansyl-2S,4R-Flp-hPhe-Arg-OH: m/z: MALDI-TOF [M+H] $]^{+}: 684.28$ (Found), 684.30 (Calc). Semi-Prep HPLC: Xbridge; $20-80 \%$ B over 10 min. Rt $=5.17$ min. Analytical HPLC: Proto 200; $20-80 \%$ B over $10 \mathrm{~min} . \mathrm{Rt}=5.81 \mathrm{~min}$.<smiles>CC(CN(C)C)N(C)c1cccc2c(S(=O)(=O)N3CCCC[C@H]3C(=O)N[C@@H](CCc3ccccc3)C(=O)O)cccc12</smiles> 
Compound 19 Dansyl-Pip-hPhe- $\mathrm{OH}$ : m/z: MALDI-TOF $[\mathrm{M}+\mathrm{H}]^{+}: 524.60$ (Found), 524.22 (Calc). Semi-Prep HPLC: Xbridge; $20-80 \%$ B over $10 \mathrm{~min}$. Rt = $7.60 \mathrm{~min}$. Analytical HPLC: Proto 200; 20 $-100 \%$ B over $10 \mathrm{~min} . \mathrm{Rt}=6.91 \mathrm{~min}$.

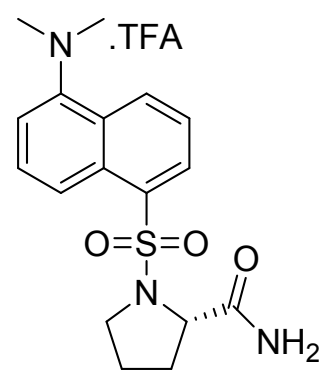

Compound 20* Dansyl-Pro- $\mathrm{NH}_{2}: \mathrm{m} / \mathrm{z}$ : MALDI-TOF $[\mathrm{M}+\mathrm{H}]^{+}: 348.58$ (Found), 348.14 (Calc). ${ }^{1} \mathrm{H}$ NMR (400 MHz $\left.\mathrm{CD}_{3} \mathrm{OD}\right) \delta 8.77(1 \mathrm{H}, \mathrm{d}, J=8.5 \mathrm{~Hz}), 8.59(1 \mathrm{H}, \mathrm{d}, J=8.5 \mathrm{~Hz}), 8.33(1 \mathrm{H}, \mathrm{d}, J=7.5$ $\mathrm{Hz}), 7.77-7.70(2 \mathrm{H}, \mathrm{m}), 7.62(1 \mathrm{H}, \mathrm{d}, J=7.5 \mathrm{~Hz}), 4.34(1 \mathrm{H}, \mathrm{d}, J=6.0 \mathrm{~Hz}), 3.57-3.52(1 \mathrm{H}, \mathrm{m}), 3.44$ $-3.37(1 \mathrm{H}, \mathrm{m}), 3.14(6 \mathrm{H}, \mathrm{s}), 2.00-1.87(3 \mathrm{H}, \mathrm{m}), 1.75-1.68(1 \mathrm{H}, \mathrm{m}) .{ }^{13} \mathrm{C}\left(101 \mathrm{MH}_{\mathrm{Z}}, \mathrm{CD}_{3} \mathrm{OD}\right) \delta$ $175.9,134.1,130.4,130.3,128.7,128.4,127.8,124.5,122.5,116.8,61.5,48.9,45.2,31.1,24.3$.

*Compound 20 was used without HPLC purification.

\section{References}

1. Chambraud, B.; Rouvière-Fourmy, N.; Radanyi, C.; Hsiao, K.; Peattie, D.A.; Livingston, D.J.; Baulieu, E.E. Overexpression of P59-HBI, Full Length and Domains, and Characterization of PPlase Activity. Biochem. Biophys. Res. Comm., 1993, 196, 160. 